\title{
Numerical continuation of bound and resonant states of the two-channel Schrödinger equation
}

\author{
P. Kłosiewicz, ${ }^{*}$ W. Vanroose, ${ }^{\dagger}$ and J. Broeckhove ${ }^{\ddagger}$ \\ Department of Mathematics and Computer Science, University of Antwerp, Middelheimlaan 1, BE-2020 Antwerp, Belgium
}

(Received 12 September 2011; published 24 January 2012)

\begin{abstract}
Resonant solutions of the quantum Schrödinger equation occur at complex energies where the $S$ matrix becomes singular. Knowledge of such resonances is important in the study of the underlying physical system. Often the Schrödinger equation depends on some parameter and one is interested in following the path of the resonances in the complex energy plane as the parameter changes. This is particularly true in coupled-channel systems where the resonant behavior is highly influenced by the strength of the channel coupling, the energy separation of the channels, and other factors. In previous work it was shown that numerical continuation, a technique familiar in the study of dynamical systems, can be brought to bear on the problem of following the resonance path in one-dimensional problems [J. Broeckhove, P. Klosiewicz, and W. Vanroose, J. Comput. Appl. Math. 234, 1238 (2010).] and multichannel problems without energy separation between the channels [P. Kłosiewicz, J. Broeckhove, and W. Vanroose, Commun. Comput. Phys.11, 435 (2012).]. A regularization can be defined that eliminates coalescing poles and zeros that appear in the $S$ matrix at the origin due to symmetries. Following the zeros of this regularized function then traces the resonance path. In this work we show that this approach can be extended to channels with energy separation, albeit limited to two channels. The issue here is that the energy separation introduces branch cuts in the complex energy domain that need to be eliminated with a so-called uniformization. We demonstrate that the resulting approach is suitable for investigating resonances in two-channel systems and provide an extensive example.
\end{abstract}

DOI: 10.1103/PhysRevA.85.012709

PACS number(s): 03.65.Nk, 02.30.Oz, 02.60.Cb, 03.65.Ge

\section{INTRODUCTION}

The presence of resonances can drastically increase the yield of many quantum mechanical reactions [1]. A resonance is an intermediate state that is formed when reagents collide with an appropriate energy and form an excited complex that decays into reactants or products. In molecular reactions these intermediate states are often electronic excited states. In the Born-Oppenheimer picture the molecular dynamics temporarily follows the electronic potential energy surface formed by the resonant state.

This picture forms the basis of state-of-the-art $a b$ initio calculations where, first, the position and lifetime of the resonance is determined using electronic scattering calculations with the nuclei fixed in space. This calculation needs to be repeated for every possible configuration of the nuclear positions probed by the chemical reactions and this results in the resonant potential energy surface. In the second step, the nuclear dynamics is simulated on the resonant potential energy surface that leads to the reactants. This approach has been successfully used to calculate the yields of processes that are mediated by a resonant state such as dissociative electron attachment to the water molecule [2-4] and vibrational excitation of carbon dioxide $[5,6]$.

This work focuses on the first step outlined above, where the potential curves are calculated as a function of the nuclear degrees of freedom. We will study a model consisting of a coupled Schrödinger equation and construct, in an automatic way, the potential curve of a resonance that becomes bound as the parameters in the equation are varied. The method tracks the resonances accurately, even in parameter ranges where the

\footnotetext{
*przemyslaw.klosiewicz@ua.ac.be

†wim.vanroose@ua.ac.be

†jan.broeckhove@ua.ac.be
}

resonance is too broad to be followed with traditional methods such as complex scaling [7].

In this article a resonance energy is defined as the complex energy at which the $S$ matrix has a pole, a common definition in the literature $[8,9]$. The real part of the pole position defines the physical resonance energy and the imaginary part defines the resonance decay width (or inverse lifetime). The advantage of this approach is that a bound state energy is also a pole of the $S$ matrix, albeit with zero decay width, that is, infinite lifetime. As such, bound states and resonances are both treated in the same way which makes it more convenient to trace states when a problem parameter changes and resonant states become bound or vice versa. The poles of the $S$ matrix will be found by a Newton iteration applied to a function that is proportional to the Jost function.

We aim to trace these states with numerical continuation. It is a technique that has found widespread application especially in the dynamical systems community. Assume one is given a solution $\left(u_{0}, \lambda_{0}\right)$ of a set of $n$ nonlinear equations $F(u, \lambda)=0$, where $F: \mathbb{R}^{n+1} \rightarrow \mathbb{R}^{n}$. A second solution $\left(u_{1}, \lambda_{1}\right)$ is then constructed numerically by applying a predictor-corrector scheme. Repeated application constructs an approximation of the implicitly defined solution set $u(\lambda)$ of $F$. One of the wellknown numerical continuation techniques is pseudo-arclength continuation proposed by Keller [10]. It has been implemented in computer programs, for example, AUTO [11,12], LOCA [13], and other numerical continuation libraries. Because the corrector step in these methods is based on Newton iterations the derivatives of the function $F$ should be Lipschitz continuous to guarantee fast convergence.

A traditional method to find the resonance position and width is complex scaling [7]. In this method, one applies a complex scaling transformation $r \rightarrow r^{i \theta}$ to the reaction coordinate. This turns the resonance wave function into 
a square integrable function. After the transformation the resonant state is then part of the discrete spectrum of the Hamiltonian. The method and its exterior variant (ECS), which has the advantage that it leaves the interactions in the inner region unchanged, have been successfully applied to find resonances in molecular systems such as HCO [14], NeICL [15], and in many other examples in atomic, molecular, and nuclear physics.

Complex scaling, however, has its limitations. First, in numerical calculations the resonance position depends slightly on the choice of the rotation angle $\theta$. This is documented for example in [7]. A second limitation is that only resonant states in a limited region in the complex energy plane can be found, in particular a pie slice between the continuum spectrum, which is rotated $2 \theta$ downward from the real axis, and the real axis. Virtual states for example, have a purely imaginary wave number and lie outside this region. As such they are hard to find with this method.

Since a resonance often transforms, as the parameters of the system change, very shortly, into a virtual state before it becomes a bound state, it is necessary to have a mathematical description that can handle these virtual states. Such a method will make it possible to understand how resonances and bound states are connected.

This paper investigates the application of numerical continuation to trace resonant states in a coupled-channel Schrödinger equation as the parameters of the problem change. Unfortunately, the application of numerical continuation to trace resonant states is not without challenges. For Newton's method to work efficiently it requires a function whose derivatives are Lipschitz continuous [16]. The $S$ matrix does not satisfy these smoothness conditions. In particular when a resonance makes the transition to a bound state, a pole and a zero of the $S$ matrix meet and straightforward application of numerical continuation fails to trace the zero of $1 / \mathrm{S}$.

In [17] it was found for one-dimensional quantum systems that it is possible to apply the continuation to a regularized function derived from the $S$ matrix because that function does satisfy the necessary smoothness conditions. For several realistic potentials the resonances were tracked as parameters in the system were varied. The method was validated against well known benchmark problems such as the classic Nussenzveig model [18].

In [19] the method has been extended to many-channel problems where all channels have the same asymptotic energy threshold. Applications have demonstrated the viability of the approach in tracing the parameter dependence of the resonance in the system. Again the method was validated against academic model problems. However, as it stands, the method does not apply to systems where the channels have unequal energy thresholds. In this case, branch cuts occur in the complex energy plane making the method invalid.

This is a significant restriction for certain application areas, for example, most problems in molecular dynamics have such unequal energy thresholds. In this paper we will investigate this difficulty and show that in the two-channel case it can be overcome by introducing an appropriate uniformization of the complex plane. In addition, we demonstrate the numerical practice of the method using an extensive example.

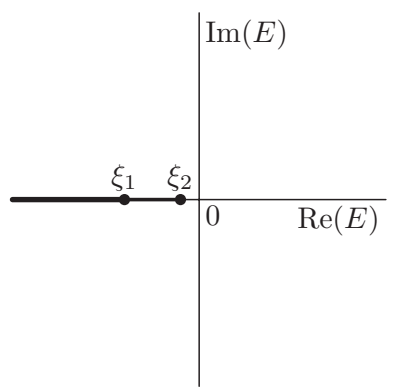

(a) With unequal thresholds, branch cuts at $\left(-\infty, \xi_{i}\right]$.

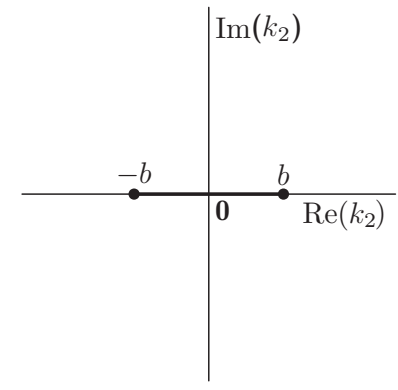

(b) Even in the $k_{1}$ or $k_{2}$-plane there is a branch cut at $[-b, b]$
FIG. 1. Branch cuts of $\mathbf{S}$ in $E$ and $k$ representation for a two channel system.

The main result of the paper is the extension of the applicability of numerical continuation to track resonant states in coupled-channel problems with unequal thresholds. Another result is the comparison with ECS that highlights the advantages of our approach. Although in this paper the method is applied to a rather academic problem, we believe it is an important step in the development of a robust technique that can automatically generate potential curves for real molecules.

We proceed as follows: In Sec. II we formulate the equations of interest in the context of quantum mechanical systems. Section III describes the so-called scattering matrix or $S$ matrix, its relation to resonances and bound states, as well as the complex geometries that occur in the $S$ matrix of coupled-channel systems. Section IV gives a brief overview of the background and applications of numerical continuation techniques. Section V outlines the details of our implementation of the methods described in the preceding sections. In Sec. VI we present an excerpt of the results we have obtained and compare with the ECS method and in Sec. VII we give an outlook for possible applications and future studies.

\section{COUPLED-CHANNEL SCHRÖDINGER EQUATION}

The time-independent Schrödinger equation for $N$ coupled channels with a spherically symmetric potential reads

$$
\begin{aligned}
& {\left[-\frac{1}{2 \mu} \frac{d^{2}}{d r^{2}} \mathbf{I}+\frac{\mathbf{L}(\mathbf{L}+\mathbf{I})}{2 \mu r^{2}}+\mathbf{V}(r, \lambda)+\Xi\right] \Psi(r ; E, \lambda)} \\
& \quad=E \Psi(r ; E, \lambda),
\end{aligned}
$$

where $\left(r \in \mathbb{R}^{+}\right)$is the radial coordinate, $\mathbf{I}$ is the $N \times N$ identity matrix, $\Xi$ is the diagonal matrix of channel thresholds $\xi_{i}, \mathbf{L}$ is the diagonal matrix of channel angular momenta $l_{i}, \mathbf{V}(r, \lambda)$ is the matrix of channel and coupling potentials $V_{i j}(r, \lambda)$, which depend on a problem parameter $\lambda \in \mathbb{R}$, and finally $\Psi(r)$ is the matrix of channel wave functions. Depending on the properties of the potential matrix $\mathbf{V}(r, \lambda)$ at infinity the behavior of the solutions $\Psi(r)$ will differ significantly. We assume so called short range interactions: $V_{i j}(r)$ vanishes faster than $r^{-3}$ as $r \rightarrow \infty$ and is less singular than $r^{-2}$ in the origin $r=0$, see also [9].

Given homogeneous Dirichlet boundary conditions at $r=$ 0 and an incoming plane wave, the asymptotic $(r \rightarrow \infty)$ 
solutions behave as

$$
\Psi(r ; E, \lambda)=\frac{i}{2}\left[\hat{\mathbf{h}}_{L}^{-}(\mathbf{K} r)-\hat{\mathbf{h}}_{L}^{+}(\mathbf{K} r) \mathbf{K}^{-\frac{1}{2}} \mathbf{S}(E, \lambda) \mathbf{K}^{\frac{1}{2}}\right],
$$

where $\mathbf{K}=\sqrt{2 \mu(E \mathbf{I}-\Xi)}$ is the matrix of channel momenta $k_{i}=\sqrt{2 \mu\left(E-\xi_{i}\right)}$ and

$$
\hat{\mathbf{h}}_{L}^{ \pm}(\mathbf{K} r)=\left(\begin{array}{lll}
\hat{h}_{l_{1}}^{ \pm}\left(k_{1} r\right) & & \\
& \ddots & \\
& & \hat{h}_{l_{N}}^{ \pm}\left(k_{N} r\right)
\end{array}\right)
$$

is the matrix of spherical Riccati-Hankel functions associated with the various channels. The first term in (2) is the partial wave expansion of the incoming plane wave, the second term represents, for each incoming partial wave, the outgoing wave in all the channels. The matrix $\mathbf{S}(E, \lambda)$ is the so-called scattering or $S$ matrix.

Our main object of interest is $\mathbf{S}(E, \lambda)$ because it contains all the information about the scattering process. It can be obtained from the coupled-channel wave functions $\Psi$ through the expression

$$
\begin{aligned}
\mathbf{S}(E, \lambda)= & \mathbf{K}^{-\frac{1}{2}} \mathcal{W}\left[\hat{\mathbf{h}}_{L}^{-}\left(\mathbf{K} r_{0}\right), \Psi\left(r_{0} ; E, \lambda\right)\right] \\
& \times \mathcal{W}\left[\hat{\mathbf{h}}_{L}^{+}\left(\mathbf{K} r_{0}\right), \Psi\left(r_{0} ; E, \lambda\right)\right]^{-1} \mathbf{K}^{\frac{1}{2}},
\end{aligned}
$$

where the $\mathcal{W}$ stands for the Wronskian of two functions, whose usual definition

$$
\mathcal{W}[f(x), g(x)]=f(x) \frac{d g(x)}{d x}-\frac{d f(x)}{d x} g(x)
$$

is extended to matrices of functions as in [20]:

$$
\mathcal{W}[A(\mathbf{x}), B(\mathbf{x})]=A^{T}(\mathbf{x}) \frac{d B(\mathbf{x})}{d \mathbf{x}}-\frac{d A^{T}(\mathbf{x})}{d \mathbf{x}} B(\mathbf{x}),
$$

where $T$ is the transpose of a matrix, which vanishes in the specific case of expression (4).

The Wronskians in Eq. (4) are evaluated at a point $r_{0}$ outside the range of the potentials, usually near the edge of the computational domain. Therefore, given a numerical method for solving the Schrödinger equation (1), the $S$ matrix can be obtained numerically by evaluating matrix expression (4) which also involves the derivative of the wave functions. The details of our numerical implementation are given in Sec. V.

\section{EXTRACTING THE RESONANT AND BOUND STATES FROM THE $S$ MATRIX}

We are interested in solutions of Eq. (1) that correspond to the bound states and the resonances of the system. Many characterizations of these special solutions exist, yet the theoretically fundamental definition describes both bound and resonant states as an eigenstate of Eq. (1) with purely outgoing wave functions at infinity as the second boundary condition [8,9].

A consistent alternative definition for multichannel systems interprets these states as having energies $E$ for which $\operatorname{det}[\mathbf{S}(E, \lambda)]$ exhibits a pole. This is consistent with the first definition, as is easy to see when one looks at the expression for the asymptotic wave function (2). If $\operatorname{det}[\mathbf{S}(E, \lambda)]$ has a pole, then in at least one eigenchannel [i.e., the channels defined by linear transformation to diagonalize the $\mathbf{S}(E, \lambda)$ matrix]

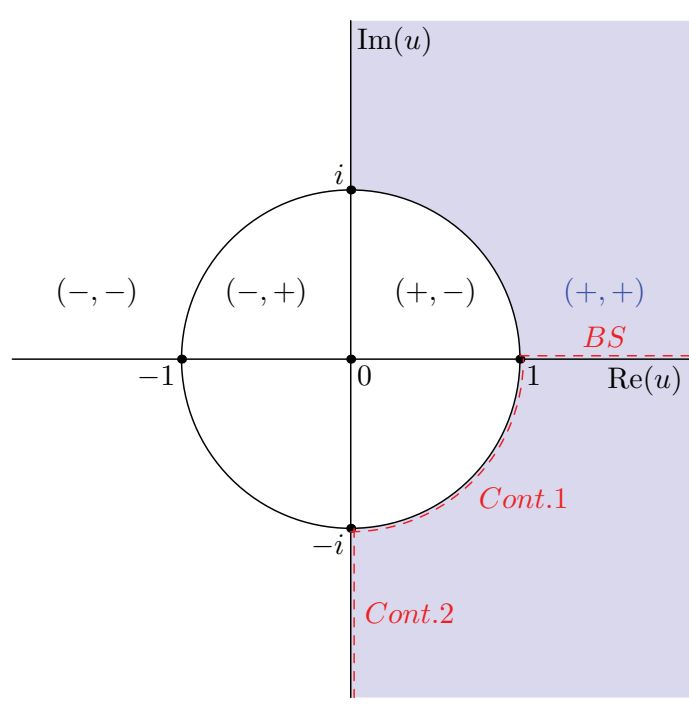

FIG. 2. (Color online) Uniformization of the four-sheeted Riemann surface of $\mathbf{S}(E)$ using the transformation (10) unravels the sheets onto four different regions of the complex $u$ plane. The blue (gray) region $(+,+)$ corresponds to the physical sheet $|u|>1, \operatorname{Re}(u)>0$. Bound states $E \in\left(-\infty, \xi_{1}\right]$ lie on $u \in[1,+\infty)$. Scattering states $E \in\left[\xi_{1}, \xi_{2}\right]$ map to $u=e^{i \theta}, \theta \in\left[-\frac{\pi}{2}, 0\right]$. Scattering states $E \in\left[\xi_{2},+\infty\right)$ map to $u \in[-i,-i \infty)$.

a resonant solution proportional to a purely outgoing wave occurs. A more formal approach relies on the introduction of Jost functions, for which we refer to the literature [8,9].

From the definition of the bound and the resonant states as a pole of $\operatorname{det}[\mathbf{S}(E, \lambda)]$ it is now possible to study the evolution of these states in terms of a changing system parameter $\lambda \in \mathbb{R}$. As $\lambda$ traverses the parameter space, the bound and resonant states change position and lifetime. It is of interest for many applications to know the explicit dependence on the parameter of choice because it leads to the resonant and bound state potential surface.

It would be straightforward to define resonance trajectories as curves in the complex $E$ plane parametrized by $\lambda$,that is, $\left\{(E, \lambda) \in \mathbb{C} \times \mathbb{R} \mid \operatorname{det}[\mathbf{S}(E, \lambda)]^{-1}=0\right\}$. Although this is theoretically correct, it is not feasible numerically because of two reasons. First, in the general case, the coupled-channel $S$ matrix is a multivalued complex function of the energy and "lives" on a multisheeted Riemann surface with branch cuts for every threshold value. Second, near threshold parameter values where bound and resonant states meet, multiple poles and zeros of the $S$ matrix coalesce, thereby destroying local smoothness properties. This is a consequence of well known symmetry properties of the $S$ matrix $[8,9]$.

The presence of branch cuts and the coalescence of zeros and poles have a strong negative impact on the convergence of the Newton iteration used in solving $\operatorname{det}[\mathbf{S}(E, \lambda)]^{-1}=0$ when calculating the resonance trajectory. A study of specific cases will give us insight in how these issues can be addressed.

\section{A. The case of $N$ channels, equal thresholds}

This case has been investigated in [19] and we briefly review the results. In Eq. (1) the matrix of channel thresholds $\Xi$ is zero 


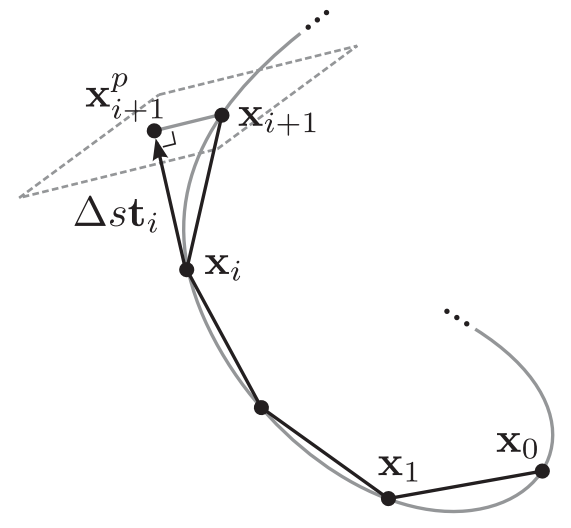

FIG. 3. Schematic representation of pseudo-arclength continuation.

(i.e., we take the threshold energy to be zero in all channels). Consequently, the matrix of channel momenta $\mathbf{K}$ becomes $k \mathbf{I}$ with $k=\sqrt{2 \mu E}$. This simplifies expression (2) to

$$
\Psi(r ; E, \lambda)=\frac{i}{2}\left[\hat{\mathbf{h}}_{L}^{-}(k r)-\hat{\mathbf{h}}_{L}^{+}(k r) \mathbf{S}(E, \lambda)\right],
$$

such that (4) becomes

$$
\begin{aligned}
\mathbf{S}(E, \lambda)= & \mathcal{W}\left[\hat{\mathbf{h}}_{L}^{-}\left(k r_{0}\right), \Psi\left(r_{0} ; E, \lambda\right)\right] \\
& \times \mathcal{W}\left[\hat{\mathbf{h}}_{L}^{+}\left(k r_{0}\right), \Psi\left(r_{0} ; E, \lambda\right)\right]^{-1} .
\end{aligned}
$$

Because $k=\sqrt{2 \mu E}$, the function $\mathbf{S}(E, \lambda)$ is defined on a two-sheet Riemann surface with a branch cut on the negative real axis $E \in \mathbb{R}^{-}$. This destroys continuity of the $S$ matrix near the branch cut and makes numerical continuation difficult. Fortunately, in this case one can express the $S$ matrix in terms of $k$ by introducing $\mathbf{S}(k, \lambda)$, a single-valued function of $k$. Afterward, the results can be easily translated back to the complex $E$ plane with $E=\frac{k^{2}}{2 \mu}$. In mathematical terms such a procedure of reparametrization of a multivalued complex function to a single-valued function is referred to as a uniformization.

Another issue that has to be dealt with is the coalescence of poles and zeros near the $k=0$ threshold value. This can be circumvented by effecting the numerical continuation on the function

$$
\begin{aligned}
F(k, \lambda) & =\operatorname{det}\left\{\mathcal{K}[\mathbf{S}(k, \lambda)-\mathbf{I}]^{-1}\right\} \\
& =\left(\prod k^{2 l_{i}+1}\right) / \operatorname{det}[\mathbf{S}(k, \lambda)-\mathbf{I}],
\end{aligned}
$$

instead of directly on the $S$ matrix where $(\mathcal{K})_{i i}=k^{2 l_{i}+1}$. In [19] it is shown that this procedure does not introduce false solutions, that is, the zeros of $F$ are precisely the poles of $\mathbf{S}$, and that it eliminates any singularities at $k=0$. This is an extension of a similar result for the one-dimensional, singlechannel systems case $[17,21]$.

\section{B. The case of two channels, unequal thresholds}

To highlight the difficulties that arise in the case of unequal thresholds, we focus on a two channel model. Each threshold $\xi_{i}$ introduces a separate branch cut $\left(-\infty, \xi_{i}\right]$, see Fig. 1(a).
Reparametrization of $\mathbf{S}$ in terms of either of the channel momenta $k_{i}$ does not provide a viable uniformization. In each of the $k_{1}$ or $k_{2}$ planes there is a branch cut at $[-b, b]$ where $b=\sqrt{2 \mu\left(\xi_{2}-\xi_{1}\right)}$ (without loss of generality we take $\xi_{1}<\xi_{2}$ ), as illustrated in Fig. 1(b). The cut disappears only when the thresholds coincide.

For two channel systems a uniformization exists, given in [8] and modified slightly in [20]. One introduces $u \in \mathbb{C}$ such that

$$
E(u)=\frac{\xi_{1}+\xi_{2}}{2}-\left|\frac{\xi_{2}-\xi_{1}}{2}\right| \frac{1+u^{4}}{2 u^{2}} .
$$

The corresponding expressions for channel momenta $k_{1}$ and $k_{2}$ are

$$
\begin{aligned}
& k_{1}(u)=i \sqrt{\mu \frac{\xi_{2}-\xi_{1}}{2}} \frac{u^{2}-1}{u}, \\
& k_{2}(u)=i \sqrt{\mu \frac{\xi_{2}-\xi_{1}}{2}} \frac{u^{2}+1}{u},
\end{aligned}
$$

and the $2 \times 2 S$ matrix is now explicitly written as

$$
\begin{aligned}
\mathbf{S}(u, \lambda)= & \left(\begin{array}{cc}
k_{1}(u) & 0 \\
0 & k_{2}(u)
\end{array}\right)^{-\frac{1}{2}} \mathbf{W}_{-}(u, \lambda) \\
& \times\left[\mathbf{W}_{+}(u, \lambda)\right]^{-1}\left(\begin{array}{cc}
k_{1}(u) & 0 \\
0 & k_{2}(u)
\end{array}\right)^{\frac{1}{2}},
\end{aligned}
$$

where

$$
\begin{aligned}
\mathbf{W}_{ \pm}(u, \lambda)= & \left(\begin{array}{cc}
\hat{h}_{l_{1}}^{ \pm}\left(k_{1}(u) r_{0}\right) & 0 \\
0 & \hat{h}_{l_{2}}^{ \pm}\left(k_{2}(u) r_{0}\right)
\end{array}\right) \\
& \times\left(\begin{array}{cc}
\frac{d}{d r} \psi_{11}\left(r_{0} ; E(u), \lambda\right) & \frac{d}{d r} \psi_{12}\left(r_{0} ; E(u), \lambda\right) \\
\frac{d}{d r} \psi_{21}\left(r_{0} ; E(u), \lambda\right) & \frac{d}{d r} \psi_{22}\left(r_{0} ; E(u), \lambda\right)
\end{array}\right) \\
& -\left(\begin{array}{cc}
\frac{d}{d r} \hat{h}_{l_{1}}^{ \pm}\left(k_{1}(u) r_{0}\right) & 0 \\
0 & \frac{d}{d r} \hat{h}_{l_{2}}^{ \pm}\left(k_{2}(u) r_{0}\right)
\end{array}\right) \\
& \times\left(\begin{array}{cc}
\psi_{11}\left(r_{0} ; E(u), \lambda\right) & \psi_{12}\left(r_{0} ; E(u), \lambda\right) \\
\psi_{21}\left(r_{0} ; E(u), \lambda\right) & \psi_{22}\left(r_{0} ; E(u), \lambda\right)
\end{array}\right),
\end{aligned}
$$

where $\psi_{i j}\left(r_{0} ; E(u), \lambda\right)$ stands for the $(i, j)$ th element of the matrix wave function $\Psi$ calculated for a specific parameter $\lambda$ and evaluated at a point $r_{0}$.

The effect of this uniformization can be visualized by looking at the different parts of the $u$ plane and how they map on the $E$ plane as illustrated in Fig. 2. Four regions $( \pm, \pm)$ in the $u$ plane can be distinguished. They are separated by the imaginary axis and the unit circle and correspond to the four different sheets of $\mathbf{S}(E, \lambda)$ in the $E$ plane. The region labeled as $(+,+)$ bounded by $|u|>1$ and $\operatorname{Re}(u)>0$ is mapped to the physical sheet. Physical bound states $E \in\left(-\infty, \xi_{1}\right]$ are located on $u \in[1,+\infty)$. The physical continuum $E \in$ $\left[\xi_{1}, \xi_{2}\right]$ maps to the quarter unit circle in the fourth quadrant $u=e^{i \theta}, \theta \in\left[-\frac{\pi}{2}, 0\right]$; whereas the physical continuum $E \in$ $\left[\xi_{2},+\infty\right)$ is mapped to $u \in[-i,-i \infty)$. A more detailed description of the different regions in the $u$ plane can be found in $[8,20]$.

Continuation paths can traverse different regions in the $u$ plane, and as such, different sheets in the complex energy plane. The focus of this work is concentrated on finding these 


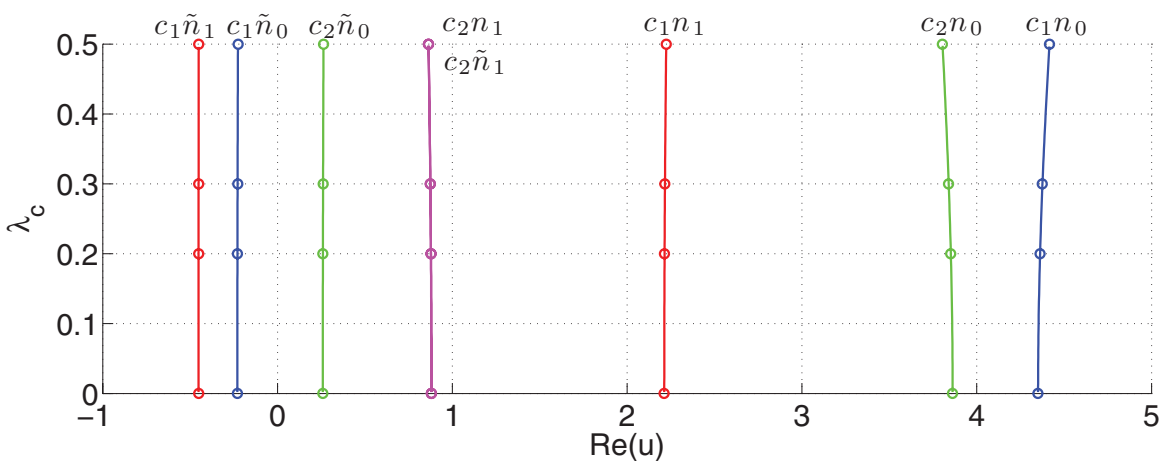

(a) $\operatorname{Re}(u) \times \lambda_{c}$ projection

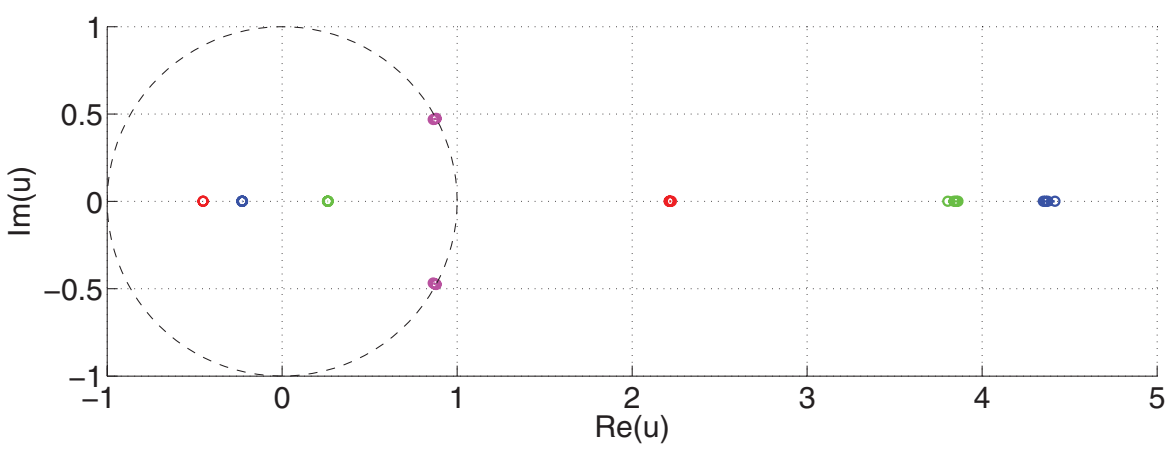

(c) $\operatorname{Re}(u) \times \operatorname{Im}(u)$ projection

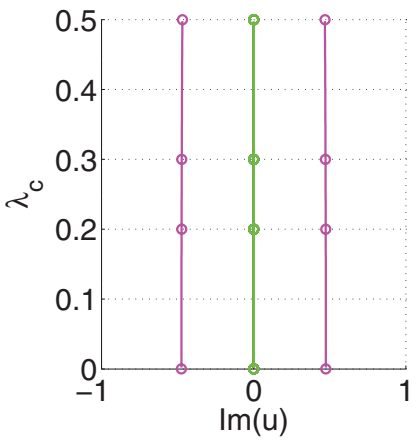

(b) $\operatorname{Im}(u) \times \lambda_{c}$ projection

FIG. 4. (Color online) Continuation paths of bound states of the example system in terms of increasing channel coupling strength $\lambda_{c}$ projected on three different planes. As the coupling strength increases, slight repulsion of the states in both channels can be observed. Circled values indicate starting points used for continuation in channel strengths $\lambda_{i}$. The values of these points are given in Table I. In (c) the dashed line represents the unit circle.

trajectories, independently of their precise physical meaning. The results we obtain are presented in the $u$ plane. However, they can be translated to the complex energy plane if one is interested in physically observable quantities.

Note that this uniformization is strictly limited to a two channel case. A similar procedure for three channel systems is much more involved, as indicated in [8]. To the knowledge of the authors there is no generalization for $N$ channels. For a thorough discussion of the analytical properties of the $S$ matrix and related functions in many-channel problems we refer to $[8,9]$.

Having established a feasible way of extracting the $S$ matrix for a specific $u \in \mathbb{C}$, we are still faced with the problem of coalescing poles and zeros as discussed in the previous case of equal thresholds. Fortunately a straightforward extension of the regularization procedure (9) can be applied. Similarly, we create the function

$$
\begin{aligned}
F(u, \lambda) & =\operatorname{det}\left\{\mathcal{K}(u)[\mathbf{S}(u, \lambda)-\mathbf{I}]^{-1}\right\} \\
& =\left[\prod_{i=1}^{N} k_{i}^{2 l_{i}+1}(u)\right] / \operatorname{det}[\mathbf{S}(u, \lambda)-\mathbf{I}],
\end{aligned}
$$

which in our case reduces to

$$
F(u, \lambda)=\frac{k_{1}^{2 l_{1}+1}(u) k_{2}^{2 l_{2}+1}(u)}{\operatorname{det}\left[\mathbf{S}(u, \lambda)-\mathbf{I}_{2}\right]} .
$$

This is the function whose solution set will be approximated through numerical continuation to obtain the trajectories of the resonant $S$-matrix poles.

\section{NUMERICAL CONTINUATION OF RESONANCES}

In the previous section we have identified a resonance trajectory starting at $\left(E_{0}, \lambda_{0}\right)$ as the implicitly defined curve $E=E(\lambda)$ with

$$
F(E(\lambda), \lambda)=0, \quad E\left(\lambda_{0}\right)=E_{0},
$$

where the function $F$ is defined in Eq. (16). We are now interested in constructing such trajectories automatically and robustly.

In dynamical systems similar equations arise in the study of steady states of parametrized ODEs and efficient methods have been developed to find the solution curves in terms of varying parameter values. In this context one is interested in finding the solution of an under determined system of nonlinear equations

$$
F: \mathbb{R}^{n+1} \longrightarrow \mathbb{R}^{n}: \mathbf{x}=(\mathbf{u}, \lambda) \longmapsto F(\mathbf{x}),
$$

connected to an initial point $\mathbf{x}_{0}=\left(\mathbf{u}_{0}, \lambda_{0}\right)$.

Many of these problems are computationally intensive and efficiency is a key concern in the numerical studies. In particular, the number of evaluations of the function $F$ should be kept to a minimum. In addition, the solution components often have complex geometries with intersections and bifurcations. The study of bifurcations generally involves rigorous stability analysis of the underlying solutions and is 


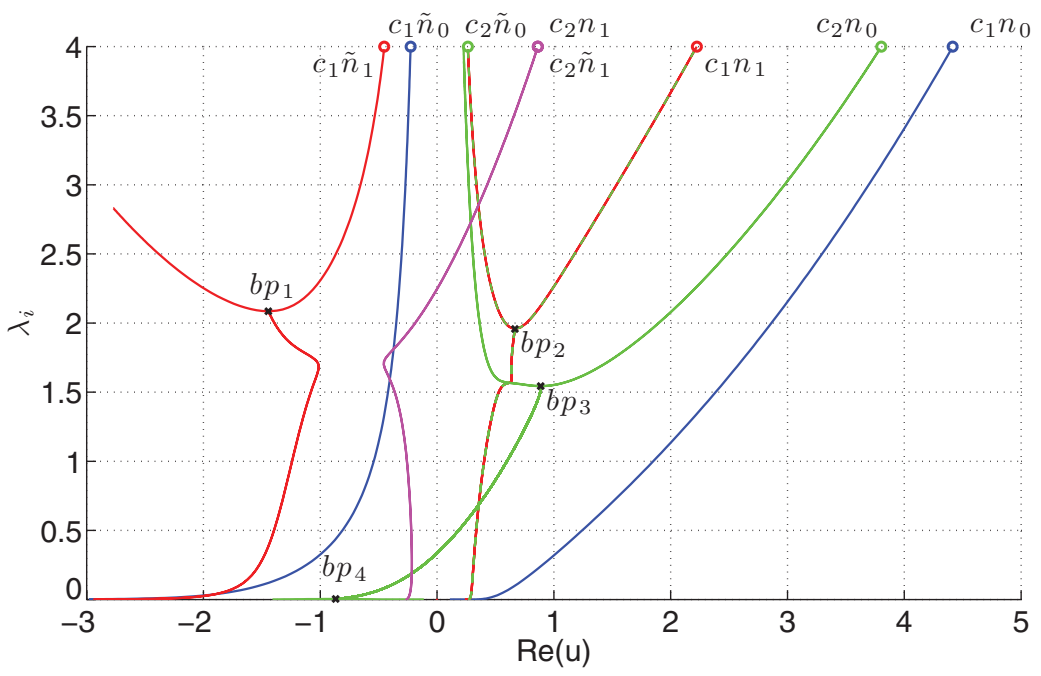

(a) $\operatorname{Re}(u) \times \lambda_{i}$

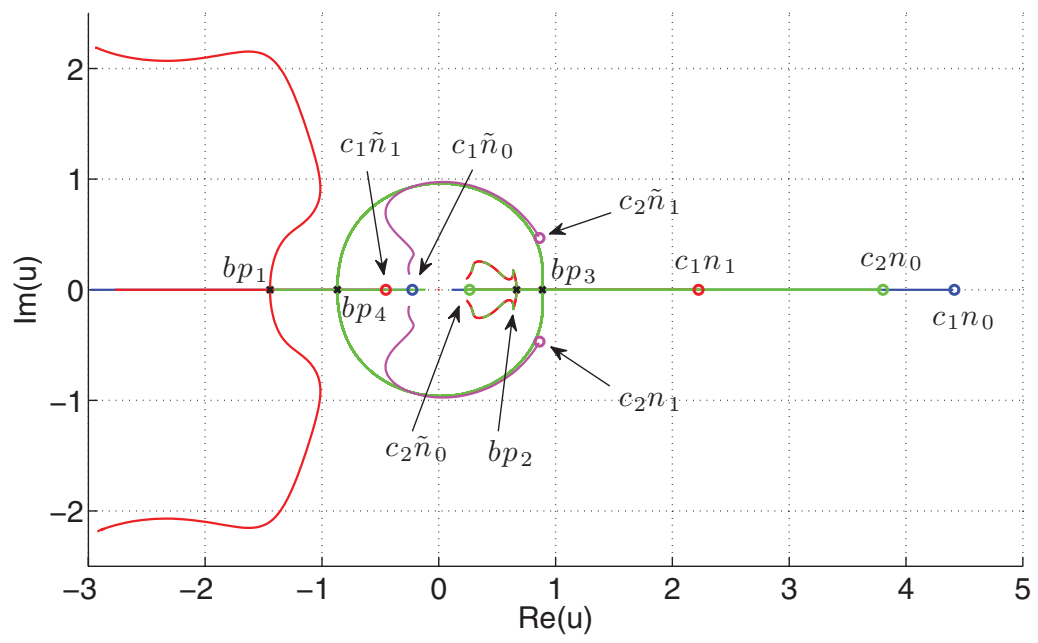

(c) $\operatorname{Re}(u) \times \operatorname{Im}(u)$

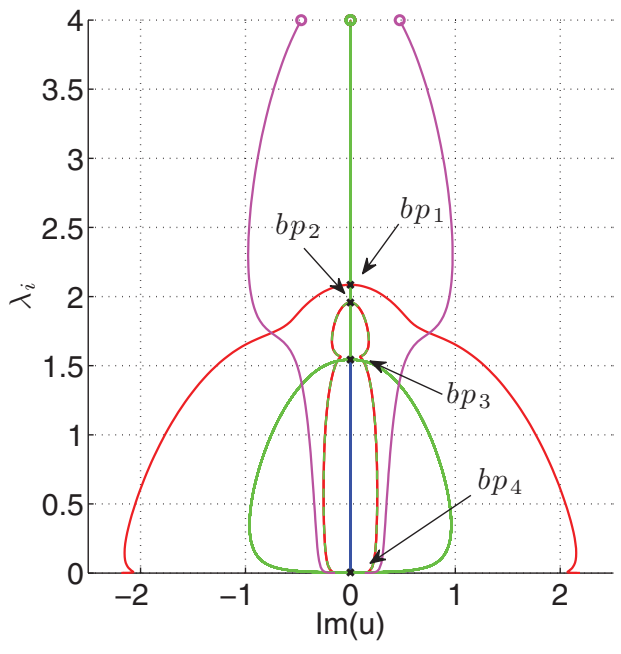

(b) $\operatorname{Im}(u) \times \lambda_{i}$

FIG. 5. (Color online) Different projections of the continuation curves $\left(\lambda_{c}=0.5\right)$. The four branch points are highlighted and labeled accordingly. Their numerical values are summarized in Table II. The connectivity graph in Fig. 6 gives a clearer overview of the associated connections.

a complicated subject on its own. In this work only the so called "simple" bifurcation points can occur. These manifest themselves as two intersecting solution branches and are characterized by the dimension of the null space of the Jacobian $F_{\mathbf{x}}\left(\mathbf{x}_{t}\right)$ being 2 in a point $\mathbf{x}_{t}$, which is called a "branch point." In many problems, however, the bifurcations are much more involved and a thorough treatise on bifurcations and stability of solutions can be found in [10,22-24].

Numerical continuation is the process of solving Eq. (18) by constructing successive approximate solutions on the path starting at the known solution $\mathbf{x}_{0}$. A good overview of these techniques can be found in [24]. There are essentially two different approaches to tracing such paths viz. piecewise linear methods and predictor-corrector methods. We will use one of the latter methods. Typically, one first makes a predictor step (Euler prediction is commonly used) that estimates the next point by following the tangent $\mathbf{t}_{i}$ to the curve at the current point $\mathbf{x}_{i}$ for a certain small distance $\Delta s$ as in $\mathbf{x}_{i+1}^{p}=\mathbf{x}_{i}+\Delta s \mathbf{t}_{i}$.
Next, a corrector step is applied to converge to a solution $\mathbf{x}_{i+1}^{p} \longrightarrow \mathbf{x}_{i+1}$. Quite often Newton iterations are used as a corrector.

One such predictor-corrector method, the one we will use in this paper, is the pseudo-arclength continuation. Its corrector step consists of Newton iterations on the system $F(\mathbf{x})=\mathbf{0}$ augmented with an additional equation that constrains the iterations to a hyper plane through $\mathbf{x}_{i+1}^{p}$ and perpendicular to the tangent $\mathbf{t}_{i}$ thereby giving the next point $\mathbf{x}_{i+1}$ on the solution curve (see Fig. 3).

A robust implementation of numerical continuation that can detect branch points and continue branching solution curves is provided by AUTO [11,12], which we have used in this work. Other well-known implementations of numerical continuation algorithms include LOCA [13] which is a part of the Trilinos framework [25], MATCONT [26] (a MATLAB implementation), and MULTIFARIO [27] which allows multiparameter continuation. 
TABLE I. Numerical values of the states in the $u$ and $E$ planes. Different states with coupling values (top to bottom) $\lambda_{c}=0, \lambda_{c}=0.2$, $\lambda_{c}=0.3$, and $\lambda_{c}=0.5$ are shown. The labels are used in the figures to make a clear distinction between states and their trajectories.

\begin{tabular}{|c|c|c|c|c|c|c|c|}
\hline$\lambda_{c}$ & Channel & State & Label & $\operatorname{Re}(u)$ & $\operatorname{Im}(u)$ & $\operatorname{Re}(E)$ & $\operatorname{Im}(E)$ \\
\hline \multirow[t]{8}{*}{0} & \multirow[t]{4}{*}{1} & \multirow[t]{2}{*}{$n_{0}$} & $c_{1} \tilde{n}_{0}$ & $-2.2983975 \times 10^{-1}$ & 0 & -2.1228484 & 0 \\
\hline & & & $c_{1} n_{0}$ & 4.3508575 & 0 & -2.1228484 & 0 \\
\hline & & \multirow[t]{2}{*}{$n_{1}$} & $c_{1} \tilde{n}_{1}$ & $-4.5199837 \times 10^{-1}$ & 0 & $-3.8737558 \times 10^{-1}$ & 0 \\
\hline & & & $c_{1} n_{1}$ & 2.2123974 & 0 & $-3.8737558 \times 10^{-1}$ & 0 \\
\hline & \multirow[t]{4}{*}{2} & \multirow[t]{2}{*}{$n_{0}$} & $c_{2} \tilde{n}_{0}$ & $2.5892712 \times 10^{-1}$ & 0 & -1.6228484 & 0 \\
\hline & & & $c_{2} n_{0}$ & 3.8620906 & 0 & -1.6228484 & 0 \\
\hline & & \multirow[t]{2}{*}{$n_{1}$} & $c_{2} \tilde{n}_{1}$ & $8.8019950 \times 10^{-1}$ & $4.7460388 \times 10^{-1}$ & $1.1262442 \times 10^{-1}$ & 0 \\
\hline & & & $c_{2} n_{1}$ & $8.8019950 \times 10^{-1}$ & $-4.7460388 \times 10^{-1}$ & $1.1262442 \times 10^{-1}$ & 0 \\
\hline \multirow[t]{8}{*}{0.2} & \multirow[t]{4}{*}{1} & \multirow[t]{2}{*}{$n_{0}$} & $c_{1} \tilde{n}_{0}$ & $-2.2923691 \times 10^{-1}$ & 0 & -2.1352756 & 0 \\
\hline & & & $c_{1} n_{0}$ & 4.3623083 & 0 & -2.1352854 & 0 \\
\hline & & \multirow[t]{2}{*}{$n_{1}$} & $c_{1} \tilde{n}_{1}$ & $-4.5179967 \times 10^{-1}$ & 0 & $-3.8789140 \times 10^{-1}$ & 0 \\
\hline & & & $c_{1} n_{1}$ & 2.2141945 & 0 & $-3.8832855 \times 10^{-1}$ & 0 \\
\hline & \multirow[t]{4}{*}{2} & \multirow[t]{2}{*}{$n_{0}$} & $c_{2} \tilde{n}_{0}$ & $2.5963744 \times 10^{-1}$ & 0 & -1.6127068 & 0 \\
\hline & & & $c_{2} n_{0}$ & 3.8517883 & 0 & -1.6129594 & 0 \\
\hline & & \multirow[t]{2}{*}{$n_{1}$} & $c_{2} \tilde{n}_{1}$ & $8.7757633 \times 10^{-1}$ & $4.7363933 \times 10^{-1}$ & $1.1278823 \times 10^{-1}$ & $1.1579542 \times 10^{-3}$ \\
\hline & & & $c_{2} n_{1}$ & $8.7757633 \times 10^{-1}$ & $-4.7363933 \times 10^{-1}$ & $1.1278823 \times 10^{-1}$ & $-1.1579542 \times 10^{-3}$ \\
\hline \multirow[t]{8}{*}{0.3} & \multirow[t]{4}{*}{1} & \multirow[t]{2}{*}{$n_{0}$} & $c_{1} \tilde{n}_{0}$ & $-2.2852083 \times 10^{-1}$ & 0 & -2.1501654 & 0 \\
\hline & & & $c_{1} n_{0}$ & 4.3759865 & 0 & -2.1501849 & 0 \\
\hline & & \multirow[t]{2}{*}{$n_{1}$} & $c_{1} \tilde{n}_{1}$ & $-4.5155161 \times 10^{-1}$ & 0 & $-3.8853641 \times 10^{-1}$ & 0 \\
\hline & & & $c_{1} n_{1}$ & 2.2164315 & 0 & $-3.8951600 \times 10^{-1}$ & 0 \\
\hline & \multirow[t]{4}{*}{2} & \multirow[t]{2}{*}{$n_{0}$} & $c_{2} \tilde{n}_{0}$ & $2.6048642 \times 10^{-1}$ & 0 & -1.6006947 & 0 \\
\hline & & & $c_{2} n_{0}$ & 3.8395472 & 0 & -1.6012444 & 0 \\
\hline & & \multirow[t]{2}{*}{$n_{1}$} & $c_{2} \tilde{n}_{1}$ & $8.7428785 \times 10^{-1}$ & $4.7241720 \times 10^{-1}$ & $1.1298423 \times 10^{-1}$ & $2.6183712 \times 10^{-3}$ \\
\hline & & & $c_{2} n_{1}$ & $8.7428785 \times 10^{-1}$ & $-4.7241720 \times 10^{-1}$ & $1.1298423 \times 10^{-1}$ & $-2.6183712 \times 10^{-3}$ \\
\hline \multirow[t]{8}{*}{0.5} & \multirow[t]{4}{*}{1} & \multirow[t]{2}{*}{$n_{0}$} & $c_{1} \tilde{n}_{0}$ & $-2.2645171 \times 10^{-1}$ & 0 & -2.1939897 & 0 \\
\hline & & & $c_{1} n_{0}$ & 4.4159879 & 0 & -2.1940286 & 0 \\
\hline & & \multirow[t]{2}{*}{$n_{1}$} & $c_{1} \tilde{n}_{1}$ & $-4.5076010 \times 10^{-1}$ & 0 & $-3.9060199 \times 10^{-1}$ & 0 \\
\hline & & & $c_{1} n_{1}$ & 2.2235200 & 0 & $-3.9328811 \times 10^{-1}$ & 0 \\
\hline & \multirow[t]{4}{*}{2} & \multirow[t]{2}{*}{$n_{0}$} & $c_{2} \tilde{n}_{0}$ & $2.6297322 \times 10^{-1}$ & 0 & -1.5661803 & 0 \\
\hline & & & $c_{2} n_{0}$ & 3.8041557 & 0 & -1.5675876 & 0 \\
\hline & & \multirow[t]{2}{*}{$n_{1}$} & $c_{2} \tilde{n}_{1}$ & $8.6368879 \times 10^{-1}$ & $4.6837873 \times 10^{-1}$ & $1.1354314 \times 10^{-1}$ & $7.3933316 \times 10^{-3}$ \\
\hline & & & $c_{2} n_{1}$ & $8.6368879 \times 10^{-1}$ & $-4.6837873 \times 10^{-1}$ & $1.1354314 \times 10^{-1}$ & $-7.3933316 \times 10^{-3}$ \\
\hline
\end{tabular}

\section{NUMERICAL IMPLEMENTATION}

Due to its ability to automatically find and follow bifurcating branches, we have opted to use AUTO for numerical continuation. Therefore the main component that has to be provided to implement methods described in previous sections is the actual code that calculates the numerical values of the function $F(u, \lambda)$, see Eq. (16) for a given $u \in \mathbb{C} \cong \mathbb{R}^{2}$ and $\lambda \in \mathbb{R}$.

We have solved Eq. (1) with a $O\left(h^{5}\right)$ renormalized Numerov method detailed in $[28,29]$. We also need the derivative of the numerical wave function at the end of the computational domain for a specified energy $E(u)$ in order to calculate the Wronskians in (13). We compute this derivative using a technique also described in [29] and which is of order $O\left(h^{4}\right)$. Finally, we need to calculate the function $F$ given by expression (16).

We emphasize that the numerical continuation results are independent of the underlying numerical solver of the Schrödinger equation. However, we have found that higher order methods for both the wave function and its derivative lead to significantly more robust continuation curves and allow for wider energy ranges. For this reason we selected the higher order renormalized Numerov method to generate results of Sec. VI.

This approach has been implemented in $\mathrm{C}++$ and is used as a driver routine by the AUTO program.

\section{EXAMPLES AND RESULTS}

As an example of the complicated geometries this method is able to cope with, we consider a coupled-channel $s$-wave $\left(l_{1}=l_{2}=0\right)$ system with Gaussian potential wells both as the channel potentials and the coupling. The $2 \times 2$ potential matrix has the elements

$$
\begin{aligned}
& V_{i i}\left(r, \lambda_{i}\right)=-\lambda_{i} e^{-\frac{r^{2}}{4}}, \\
& V_{i \neq j}\left(r, \lambda_{c}\right)=\lambda_{c} e^{-r^{2}},
\end{aligned}
$$

where $\lambda_{1}, \lambda_{2}$, and $\lambda_{c}$ denote the potential strength of the first, second, and coupling channels, respectively. The channel thresholds are chosen $\xi_{1}=0$ and $\xi_{2}=\frac{1}{2}$ and the mass is $\mu=1$. Equation (1) was solved using the previously mentioned renormalized Numerov method on the domain $r \in[0,4.8]$ with 4096 grid points. All of $\lambda_{1}, \lambda_{2}$, and $\lambda_{c}$ will be used as variable 


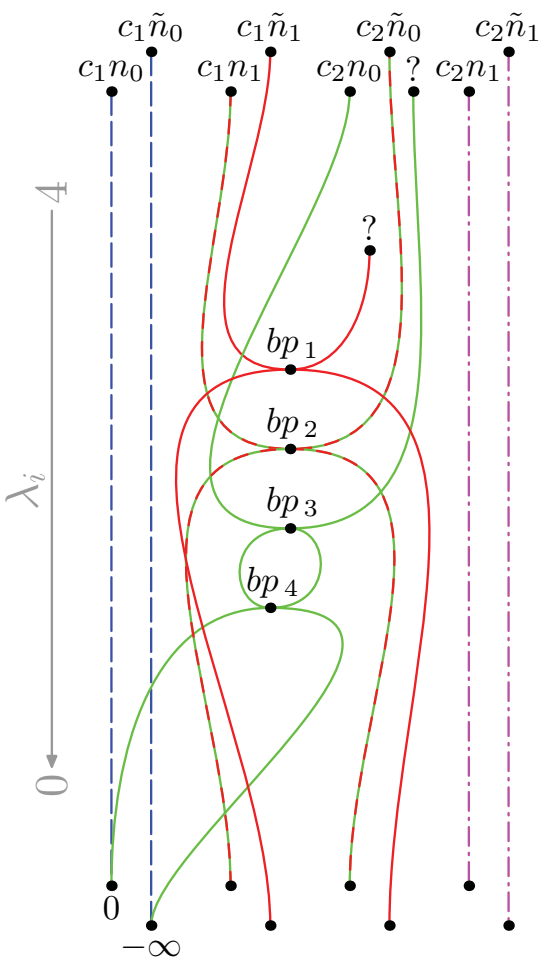

FIG. 6. (Color online) The connectivity diagram of the different continuation curves. Curves only intersect at the four points $b p_{i}$. Dashed red (dark) and green (light) lines in the center connect two states from different channels through $b p_{2}$. See Fig. 9 for a more detailed view of the connections. The end points of the curves do not play a major role and their labels are omitted in other figures as well.

system parameters. We will indicate clearly which of those are fixed and which are used as continuation parameters.

In the uncoupled case $\left(\lambda_{c}=0\right)$, setting $\lambda_{i}=4$ gives a system with two bound states in each channel whose values can be found in the upper part of Table I. Using these values as starting points we carry out the continuation in terms of increasing channel coupling $\lambda_{c}$ while keeping $\lambda_{i}=4$ fixed. The results in the $u$ plane are shown in Fig. 4. Points corresponding to coupling values $0,0.2,0.3$, and 0.5 are highlighted in the figure. Their corresponding numerical values in the $u$ and $E$ planes are summarized in Table I. Notice that for $\lambda_{c}=0$ every value of $E$ is associated with two different points in the $u$ plane which are located on different sheets. Following the paths of two such values eventually gives rise to different values in the $E$ plane. Therefore one needs to keep track of all of them. We give appropriate labels, see Table I, to distinguish different points in the $u$ plane.

While continuing in $\lambda_{c}$, only slight variations in the state's energy occur, yet the seemingly minor coupling has profound effects on the evolution of these states in terms of potential strengths $\lambda_{1}$ and $\lambda_{2}$ while keeping the coupling strength constant.

To illustrate this behavior we fix the coupling strength and use the corresponding $u$ values of the states as starting points for a continuation in terms of variations of both potential strengths $\lambda_{1}$ and $\lambda_{2}$ simultaneously. As $\lambda_{i}$ decreases, we expect

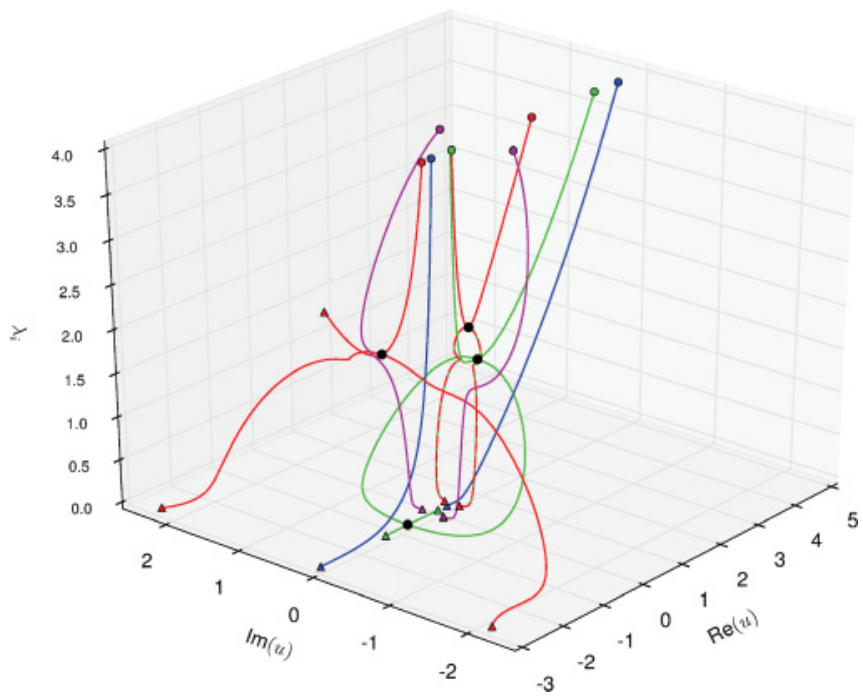

FIG. 7. (Color online) An overview of the continuation curves $\left(\lambda_{c}=0.5\right)$ shown in the full $\operatorname{Re}(u) \times \operatorname{Im}(u) \times \lambda_{i}$ space. Both start points (circles) and end points (triangles) of the curves are highlighted in respective colors. The four branch points are indicated with big black dots.

these bound states to move into the resonant regime and influence each other due to the coupling.

Although we have performed numerical continuation starting from all states for all subsequent coupling strengths from Table I, we focus on the case $\lambda_{c}=0.5$ as a highlighted example.

The resulting continuation curves are shown in Fig. 5 as projections on the planes $\operatorname{Re}(u) \times \lambda_{i}, \operatorname{Im}(u) \times \lambda_{i}$, and $\operatorname{Re}(u) \times \operatorname{Im}(u)$. Complex geometries and intersections are constructed automatically by the continuation method. An attempt to present those schematically is shown in Fig. 6 as a connectivity graph. In the range $\lambda_{i} \in[0,4]$ four branch points can be distinguished with values detailed in Table II. A three-dimensional overview of the continuation curves is shown in Fig. 7.

We have carried out the same procedure for four different values of the coupling strength using starting values summarized in Table I. As the coupling between the two channels increases, resonant trajectories undergo major qualitative changes. See Fig. 8 for a short comparison of the continuation paths projected on $\operatorname{Re}(u) \times \lambda_{i}$. The interpretation of those is beyond the scope of this paper although an important effect can be observed. In Fig. 9 a close-up view of two resonant trajectories is shown. In the uncoupled case [Fig. 9(a)] two independent trajectories of $c_{2} n_{0}$ (and $\left.c_{2} \tilde{n}_{0}\right)$ and $c_{1} n_{1}$ are shown.

TABLE II. Numerical values of the branch points of the resonant trajectories shown in Fig. 5. The coupling strength is $\lambda_{c}=0.5$.

\begin{tabular}{llrl}
\hline \hline Label & \multicolumn{1}{c}{$\lambda_{1}=\lambda_{2}$} & $u$ & $E$ \\
\hline$b p_{1}$ & 2.0852303 & -1.4443524 & $-7.0688100 \times 10^{-2}$ \\
$b p_{2}$ & 1.9571562 & $6.6636568 \times 10^{-1}$ & $-8.7009530 \times 10^{-2}$ \\
$b p_{3}$ & 1.5436785 & $8.8709701 \times 10^{-1}$ & $-7.2105286 \times 10^{-3}$ \\
$b p_{4}$ & $4.0009060 \times 10^{-3}-8.6796523 \times 10^{-1}$ & $-1.0092983 \times 10^{-2}$ \\
\hline \hline
\end{tabular}




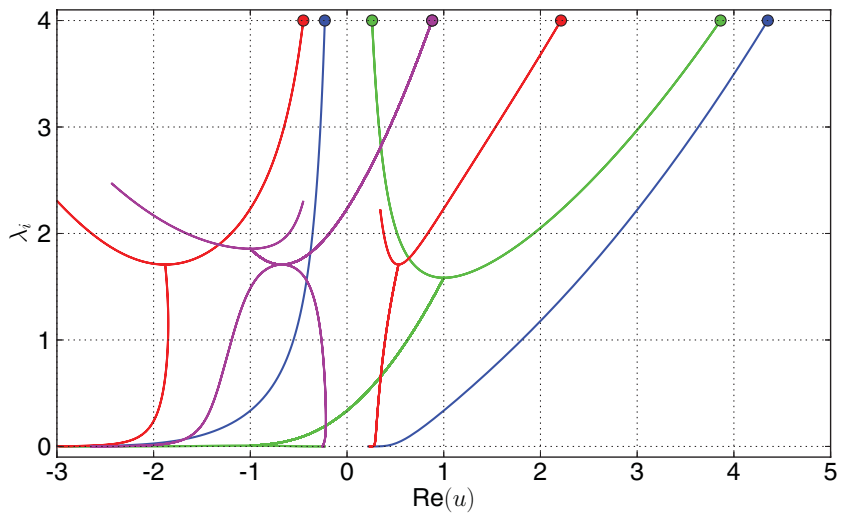

(a) $\lambda_{c}=0$

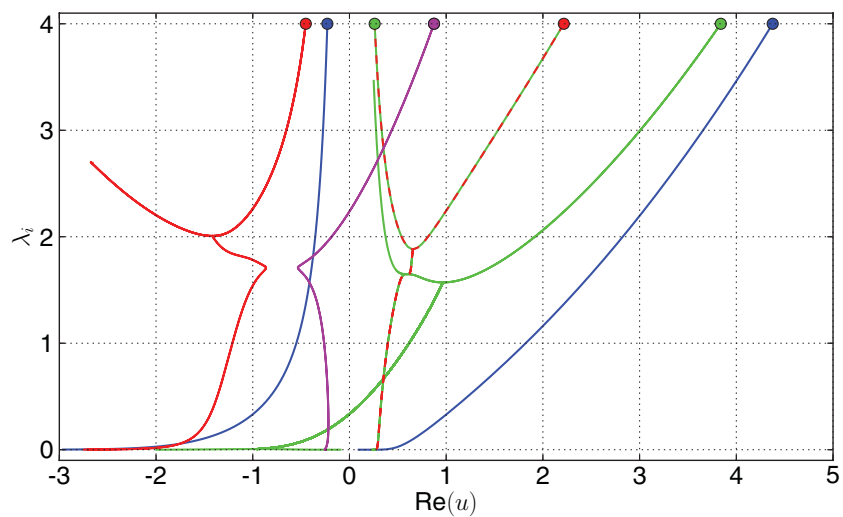

(c) $\lambda_{c}=0.3$

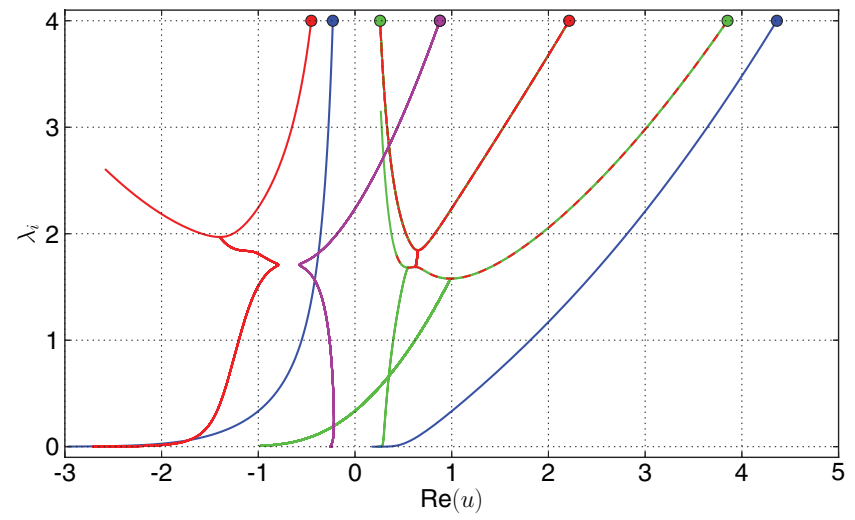

(b) $\lambda_{c}=0.2$

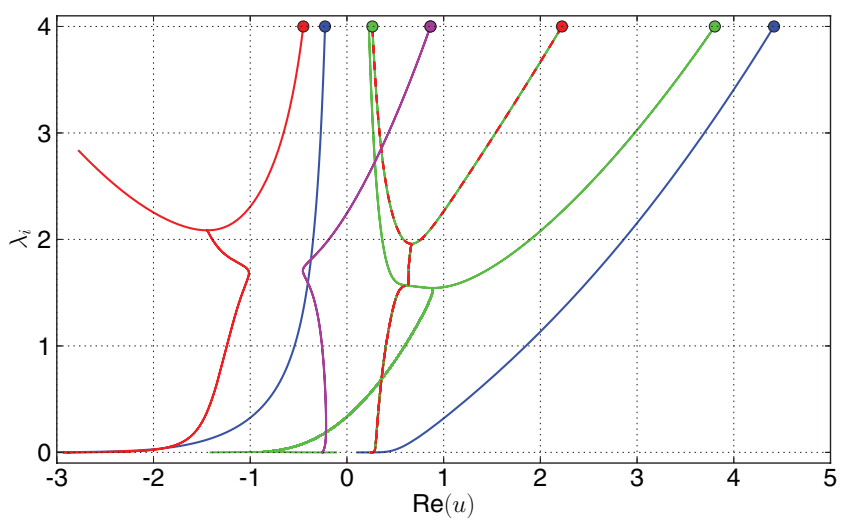

(d) $\lambda_{c}=0.5$

FIG. 8. (Color online) $\operatorname{Re}(u) \times \lambda_{i}$ projections of the continuation curves for different coupling strengths $\lambda_{c}$. Starting values are taken from Table I.

Although the green (light) and red (dark) curves intersect they do not share a branch point. The addition of a nonzero coupling in Fig. 9(b) changes this situation drastically: two more bifurcation points appear and the red and green curves are now fully connected (dashed green and red lines). As the coupling strength increases in Fig.9(c) the two additional branch points collide and disappear. For an even higher coupling [Fig. 9(d)] one can clearly see how the curves have rearranged their connections. The $c_{1} n_{1}$ state is now connected with $c_{2} \tilde{n}_{0}$ through the dashed green and red lines, whereas $c_{2} n_{0}$ shows a connection with the end point of $c_{1} n_{1}$ from the uncoupled case.

This short example highlights the ability of continuation methods to deal with subtle and complex connections.

\section{A. Comparison with exterior complex scaling}

To highlight the advantages of the numerical continuation method applied to the function $F(u, \lambda)$, we compare its results with those of a calculation with exterior complex scaling (ECS) [7]. This is done for various choices of the parameter $\lambda_{i}$. First, we translate the curves that were obtained by numerical continuation in the $u$ plane back to the $E$ plane. These are shown as the green (light) and blue (dark) curves in Fig. 10. For clarity, the colors are identical to those in the figures depicting the $u$ plane. Note that two different blue curves map to the same region in the $E$ plane. The vertical axis is the strength $\lambda_{i}$ of the channel potentials, while the bottom plane shows the complex energy of the resonant state. The real part is the proper resonance energy and the imaginary part is the inverse lifetime of the resonance. We have found both the exponentially decaying and exponentially growing states with a negative, respectively, positive imaginary energy.

A first bound state is formed as $\lambda_{i}$ increases and the potential becomes stronger. It starts out as a virtual state on the negative real energy axis and, with increasing $\lambda_{i}$, its real part increases, goes through zero and becomes negative again. This curve is shown in blue in Fig. 10.

For potential strength $\lambda_{i}$ between 0.33538 and 1.54368 we also have a resonant state with the real part of the energy between the values of the two thresholds. This state is a Feshbach resonance related to the second channel that decays through coupling with the first channel, which is open. This state also originates as a virtual state on the negative real axis at small potential strengths, similar to the state discussed above. However, it is now shifted up by 0.5 , the threshold of the second channel. Furthermore, because of the coupling to the open channel this virtual state has a finite lifetime as soon as it lies above the threshold of the open channel. When we increase the potential strength above 1.55204 this resonance becomes a second bound state, but before it becomes bound there is a bifurcation with a virtual state at parameter strength 


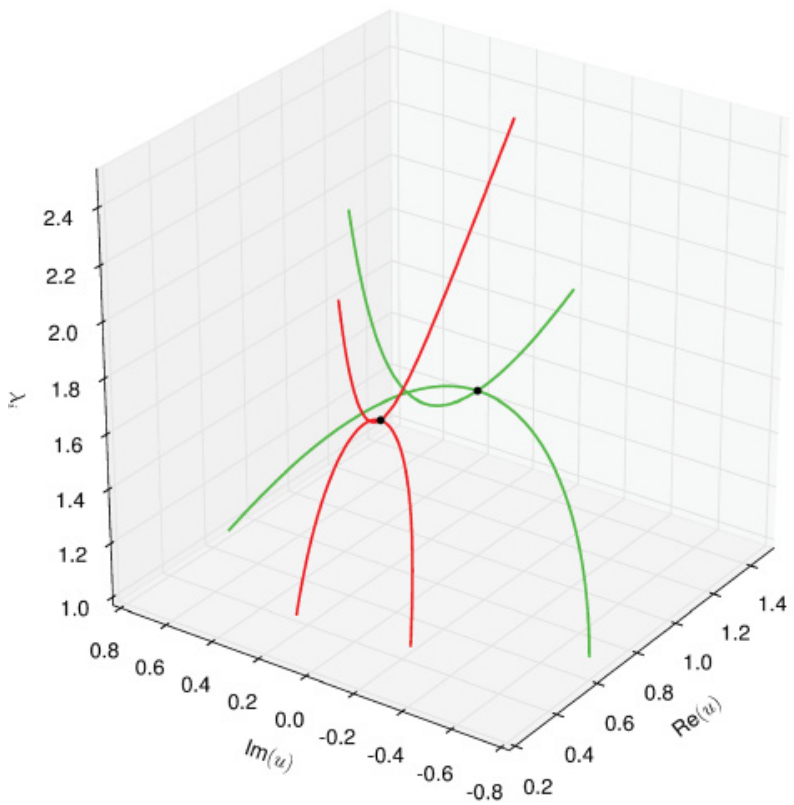

(a) $\lambda_{c}=0$

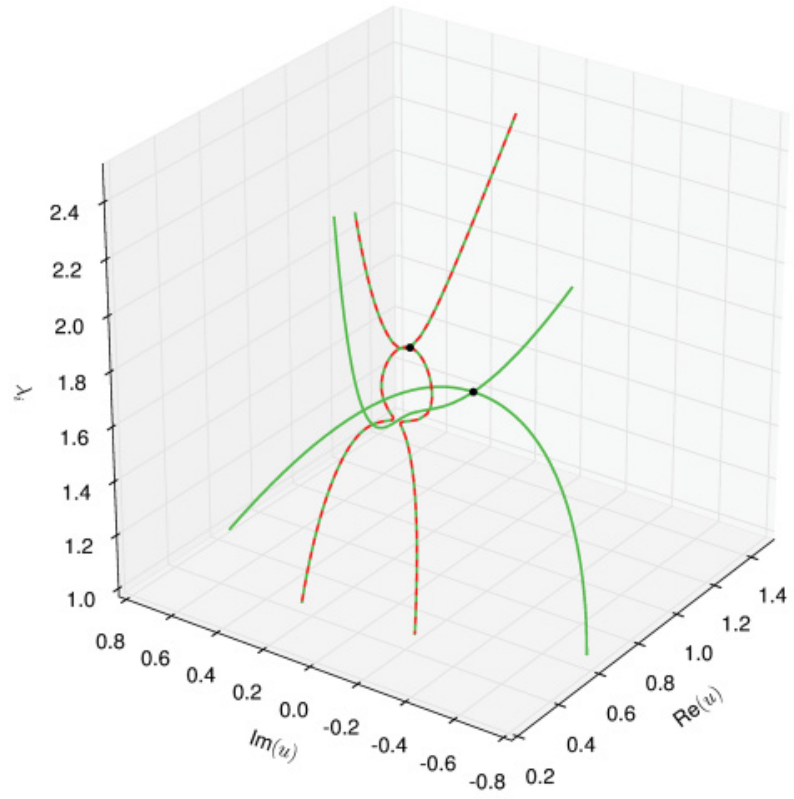

(c) $\lambda_{c}=0.3$

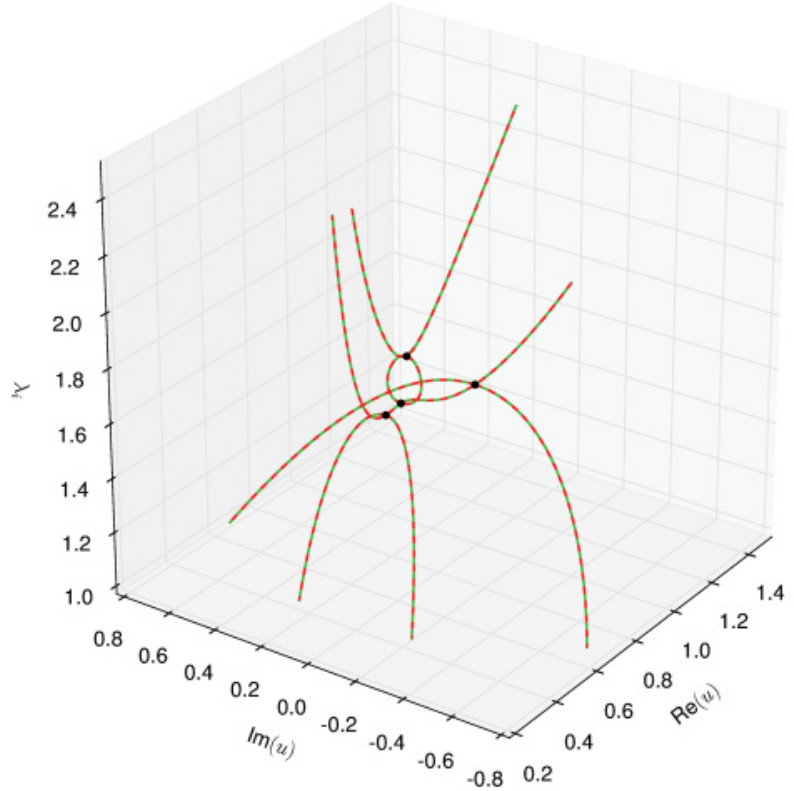

(b) $\lambda_{c}=0.2$

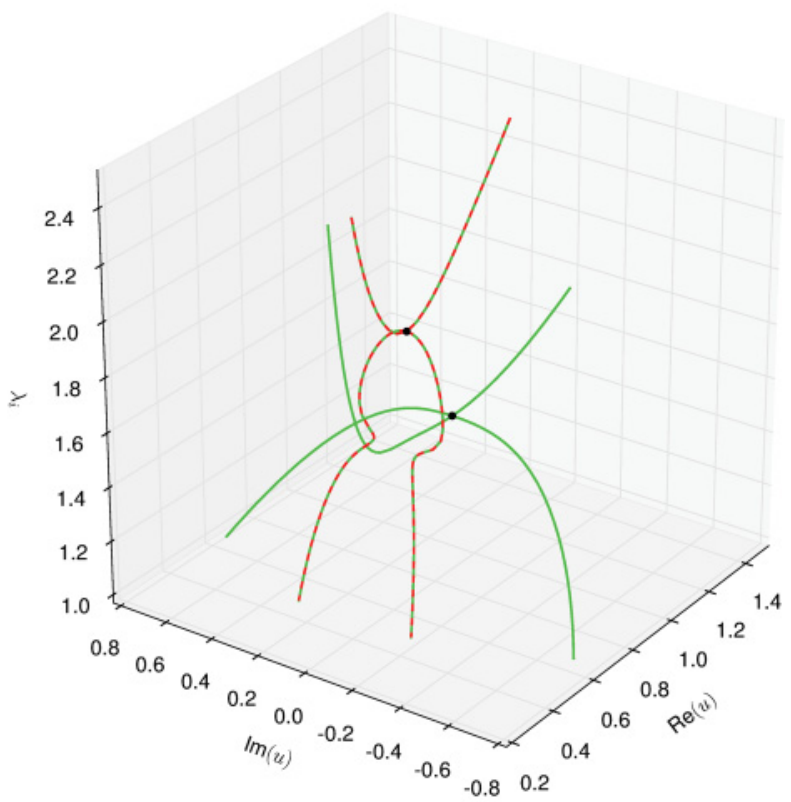

(d) $\lambda_{c}=0.5$

FIG. 9. (Color online) Closeup view of the continuation curves of $c_{2} n_{0}$ green (light), $c_{2} \tilde{n}_{0}$ green (light), and $c_{1} n_{1}$ red (dark) obtained for different values of the coupling strength $\lambda_{c}$. As the coupling strength increases an interesting effect of reordering of the connections can be observed. Initially the red (dark) and green (light) trajectories do not interact. With slight coupling, two additional branch points appear connecting both curves (dashed lines). As the coupling increases, the branch points collide and disappear disconnecting the reordered curves.

$\lambda_{i}=1.54368$. At this bifurcation point the state has negative real energy. As we further increase the strength, one of these states becomes a true bound state after passing through zero at potential strength $\lambda_{i}=1.55204$. The other point that emerges from the bifurcation is a true virtual state that moves down the negative real axis as a function of $\lambda_{i}$.

When the resonances are calculated by ECS (shown as red dots in Fig. 10), we do not resolve all these details. Especially the connections through the virtual states are missing from the picture. We discretize the two channel Hamiltonian on a finite difference grid with grid distance 0.03 and we use an exterior complex scaling transformation that starts at $r=12$ and a rotation angle of $\pi / 8$. The exterior domain extends to $\operatorname{Re}(r)=15.6$.

As expected, at zero potential strength the eigenvalues of the exterior complex scaled Hamiltonian are the discrete eigenvalues of the kinetic energy operator. These are related to standing waves on the exterior complex scaled domain. These 


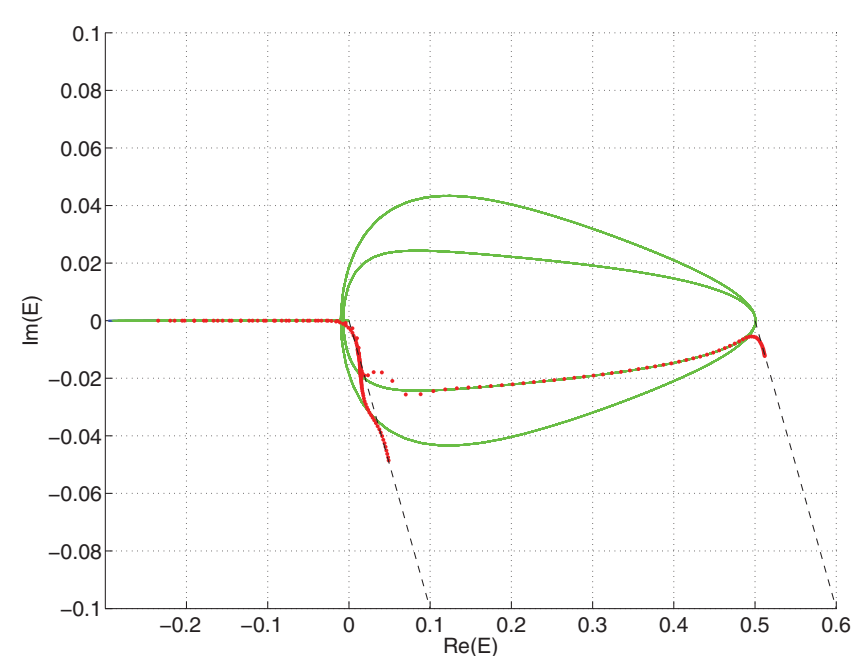

(a) $\operatorname{Re}(E) \times \operatorname{Im}(E)$ projection

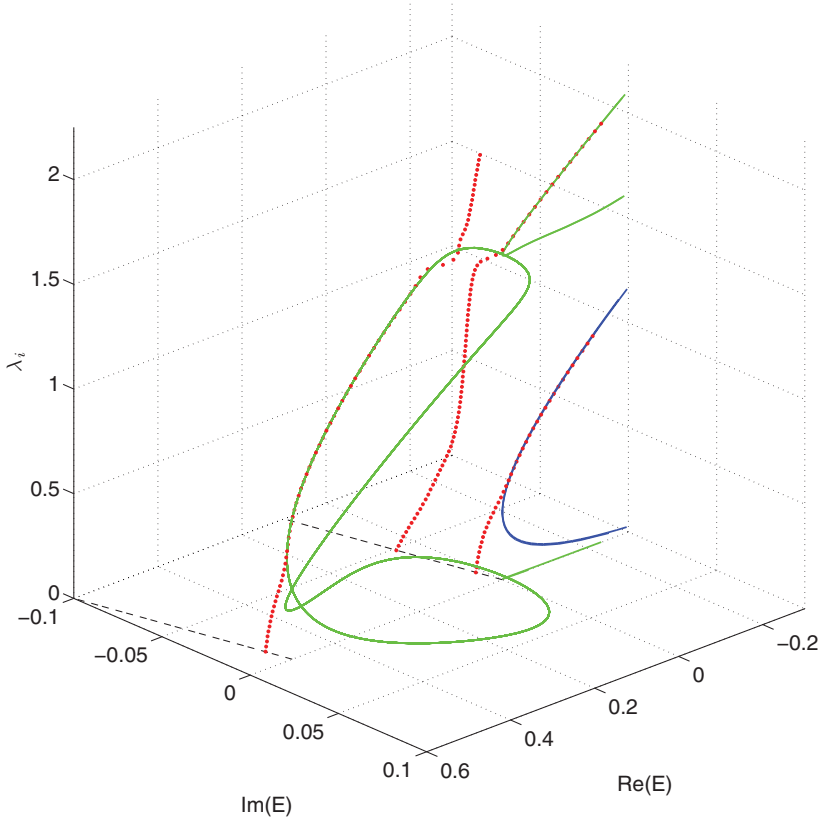

(b) $\operatorname{Re}(E) \times \operatorname{Im}(E) \times \lambda_{i}$ space

FIG. 10. (Color online) A comparison of the results of numerical continuation with the results of exterior complex scaling. The vertical axis shows the potential strength of the model problem while the real and imaginary parts of the energy are shown on the other axes. The blue (dark) and the green (light) curves are the translation of the same curves in the $u$ plane from Figs. 5 and 7. The red (dark) dots are the relevant ECS eigenvalues calculated for a range of $\lambda_{i}$ values. The results of the two methods differ significantly in the regions where a resonance becomes a bound state. Also for small potential strengths we see significant deviations since ECS cannot resolve the virtual states.

discrete continuum states will be rotated over twice the ECS rotation angle. Therefore, for each threshold we have a series of rotated eigenvalues.

As the potential strength increases, these continuum states are attracted by the potential and become bound. The first bound state is formed from the smoothest continuum state associated with the first threshold. It becomes bound at $\lambda_{i} \approx$ 0.5 . This is in contrast to the numerical continuation results where this bound state originates from a virtual state. The difference between the numerical continuation and ECS is most prominent at small potential strengths.

The second bound state in ECS is formed at potential strength $\lambda_{i} \approx 1.57$. Although this state should find its origin at zero potential strength in the smoothest continuum state of the second threshold, an avoided crossing appears instead. The curve originates from the second smoothest state of the first threshold and passes through an avoided crossing with the trajectory of the state that starts from the smoothest state of the second threshold. The former curve partly represents the bound state, whereas the latter curve partly follows the trajectory of the Feshbach resonance between potential strengths $0.46 \lesssim \lambda_{i} \lesssim 1.45$. However, as the curves are disconnected, this calculation misses the bifurcation at $\lambda_{i}=1.54368$ and the subsequent transition through a virtual state. Note that these issues occur near the line of ECS eigenvalues, starting from the first threshold going twice the ECS angle downward the complex plane. Similarly, the deviation of the trajectory around $\lambda_{i}=0.46$, as the Feshbach resonance turns into a virtual state, starts near an analogous line originating from the second threshold. These lines are drawn dashed in Fig. 10.

\section{CONCLUSIONS AND OUTLOOK}

An automatic, robust and inherently efficient method for tracking parameter dependent resonant solutions of the Schrödinger equation is desirable since they play an important role in many quantum mechanical systems. Numerical continuation based on pseudo-arclength continuation has a proven track record in being reliable and has been used in the study of various dynamical systems.

We have shown earlier that numerical continuation can be applied to track bound and resonance states in a single and coupled-channel Schrödinger equation with equal thresholds. However, when the thresholds are different additional branch cuts appear. This leads to numerical difficulties.

In this article we have shown that these numerical difficulties can be avoided when a suitable uniformization is applied. The channel momenta (i.e., the wave numbers) are then written as a function of a complex valued parameter. The numerical continuation is applied to this parameter combined with the variable parameters of the problem. Unfortunately this approach cannot be extended easily to systems with more than two channels because the uniformization procedure becomes too complex.

We have applied the method to a two channel problem with Gaussian potentials and continued in the strength of the potential for various choices of coupling strength. Several branch points were detected and the continuation automatically identified the other branches emerging from these points. Transitions between bound and resonant states are easily taken by this method. In a similar way we could 
have continued in the threshold values or any other parameters starting from any solution point.

The comparison of the results with an exterior complex scaling calculation shows significant differences in parameter ranges where the resonance transitions to a bound state. The numerical continuation results predict that this transition happens through a virtual state for the model problem. ECS however cannot resolve these virtual states and the resonance transitions directly to a bound state.

In our calculations we have detected several branch points where different states meet. All the branch points we have identified, however, are bifurcations on the negative real energy axis where two virtual states meet. This occurs when a resonance becomes bound through a scenario that was already discussed by Nussenzveig [18].

In the future we will extend the method to higher dimensional problems with multiple reaction coordinates.

Another possible future direction of research is to use two parameter continuation and automatically identify the exceptional points [30-32] where two resonances coalesce at a complex valued energy that does not necessarily lie on the real axis.
[1] H. Hotop, M.-W. Ruf, M. Allan, and I. I. Fabrikant, Adv. At. Mol. Opt. Phys. 49, 85 (2003).

[2] D. J. Haxton, Z. Zhang, C. W. McCurdy, and T. N. Rescigno, Phys. Rev. A 69, 062713 (2004).

[3] D. J. Haxton, Z. Zhang, H. D. Meyer, T. N. Rescigno, and C. W. McCurdy, Phys. Rev. A 69, 062714 (2004).

[4] D. J. Haxton, T. N. Rescigno, and C. W. McCurdy, Phys. Rev. A 75, 012711 (2007).

[5] C. W. McCurdy, W. A. Isaacs, H.-D. Meyer, and T. N. Rescigno, Phys. Rev. A 67, 042708 (2003).

[6] W. Vanroose, Z. Zhang, C. W. McCurdy, and T. N. Rescigno, Phys. Rev. Lett. 92, 053201 (2004).

[7] N. Moiseyev, Phys. Rep. 302, 211 (1998).

[8] R. G. Newton, Scattering Theory of Waves and Particles, 2nd ed., Texts and Monographs in Physics (Springer, Berlin, 1982).

[9] J. R. Taylor, Scattering Theory: The Quantum Theory of Nonrelativistic Collisions (Dover, New York, 2006).

[10] H. B. Keller, Applications of Bifurcation Theory, edited by P. H. Rabinowitz (Academic Press, New York, 1977), pp. 159-384.

[11] E. J. Doedel, Cong. Num. 30, 265 (1981).

[12] "AUTO - software for continuation and bifurcation problems in ordinary differential equations," 2007, version AUTO-07p available online.

[13] A. G. Salinger, N. M. Bou-Rabee, R. P. Pawlowski, and E. D. Wilkes, LOCA 1.1 Library Of Continuation Algorithms: Theory and Implementation Manual (Sandia National Laboratories, Albuquerque, NM, 2002).

[14] V. Ryaboy and N. Moiseyev, J. Chem. Phys. 103, 4061 (1995).

[15] N. Lipkin, N. Moiseyev, and C. Leforestier, J. Chem. Phys. 98, 1888 (1993).
[16] C. Kelley, Iterative Methods for Linear and Nonlinear Equations (SIAM, Philadelphia, 1995).

[17] J. Broeckhove, P. Klosiewicz, and W. Vanroose, J. Comput. Appl. Math. 234, 1238 (2010).

[18] H. M. Nussenzveig, Nucl. Phys. 11, 499 (1959).

[19] P. Kłosiewicz, J. Broeckhove, and W. Vanroose, Commun. Comput. Phys. 11, 435 (2012).

[20] G. V. Sitnikov and O. I. Tolstikhin, Phys. Rev. A 67, 032714 (2003).

[21] W. Vanroose, J. Broeckhove, and P. Kłosiewicz, J. Phys. B: At. Mol. Opt. Phys. 42, 044002 (2009).

[22] R. Seydel, Practical Bifurcation and Stability Analysis, From Equilibrium to Chaos (Springer, Berlin, 1994).

[23] E. J. Doedel, Numerical Continuation Methods for Dynamical Systems: Path Following and Boundary Value Problems (Springer, Dordrecht, The Netherlands, 2007), p. 51.

[24] E. L. Allgower and K. Georg, Numerical Continuation Methods An Introduction, Springer Series in Computational Mathematics, Vol. 13 (Springer, Berlin, 1990).

[25] M. A. Heroux, et al., ACM Trans. Math. Software 31, 397 (2005).

[26] A. Dhooge, W. Govaerts, Y. A. Kuznetsov, W. Mestrom, A. M. Riet, and B. Sautois, MATCONT and CL_MATCONT: Continuation Toolboxes in MATLAB (Universiteit Gent, Belgium and Utrecht University, The Netherlands, 2006).

[27] M. E. Henderson, Int. J. Bifurcation Chaos 12, 451 (2002).

[28] B. R. Johnson, J. Chem. Phys. 69, 4678 (1978).

[29] B. R. Johnson, J. Chem. Phys. 67, 4086 (1977).

[30] W. D. Heiss, Phys. Rev. E 61, 929 (2000).

[31] A. Mondragon and E. Hernandez, J. Phys. A 26, 5595 (1993).

[32] W. Vanroose, P. Van Leuven, F. Arickx, and J. Broeckhove, J. Phys. A 30, 5543 (1997). 